\title{
Application of Flexible Ureteroscope in Diagnosis and Treatment of Upper Urinary Tract Disease
}

\author{
Fan-ping MENG ${ }^{1}$, Jian-tao XIAN ${ }^{2}, \mathrm{Xu} \mathrm{ZHOU}^{3}$ and Ji GAO* \\ 1,2,3 Department of Urology, China-Japan Union Hospital Affiliated to Jilin University, \\ Changchun Jilin, 130033, China \\ * Department of Urology, China-Japan Union Hospital Affiliated to Jilin University, \\ Changchun Jilin, 130033, China
}

Keywords: Flexible ureteroscope, Upper urinary tract disease.

\begin{abstract}
Objective: To summarize the clinic experience of flexible ureteroscope in diagnosis and treatment of upper urinary tract disease. Method: From Nov 2013 to Dec 2015, 60 hematuresis cases underwent flexible ureteroscope for diagnosis and treatment of upper urinary tract. Clinical data was analyzed retrospectively. Results: All the cases were diagnosed and cured successfully in a one-time success .19 cases were diagnosed epithelium cancer, 6 cases were diagnosed non-specificity pyeloureteritis, 18 cases were diagnosed as calculus of ureter, and 8 cases were renal pelvis venous hemorrhage, no abnormality seen in 9 cases. No serious complication happened. And the time of the surgeries was 10 70 $\mathrm{min}$ and the average time was $40 \mathrm{~min}$. Hospitalization time was 3 9 days, and the average time was 4 days. Conclusion: Flexible ureteroscope can be used to diagnose and cure tumor, calculus, inflammation and other diseases of upper urinary tract, especially, to upper urinary minimal lesion. Flexible ureteroscope has an obvious advantage.

From Nov 2013 to Dec 2015, 58 hematuria patients underwent flexible ureteroscope for diagnosis and treatment of upper urinary tract diseases. Now we report as followed.
\end{abstract}

\section{Materials and Methods}

\section{General Information}

58 cases were in this group. 32 cases were male and other 26 cases were female; the average age was 42 years old.Course of these diseases were $1 \sim 2$ months, and no other clinical symptoms and complications happened. We regularly checked out the blood coagulation time, routine urine, ultrasound, cystoscopy, intravenous urography angiography and CT examination ,but we failed to diagnose. The red blood cell in the urine mainly were normal form red blood cells.

\section{Equipment}

OLYMPUS-F8 flexible ureteroscope; Sony imaging system; Ultrasonic stone crush machine; ureteral expansion tube; Guide-wire; ureteral catheter; Double J ureteral catheter.

\section{Operation Method}

We used general anesthesia .First of all, we observed the bladder and ureter openings, and tried to do the ureteral intubation, and to observe the situation of ureter. Then we removed the cystoscope, put the OLYMPUS-F8 flexible ureteroscope in bladder and observed the ureter opening of the sick side.Along the work channel of flexible ureteroscope, we put a guide-wire 
into the ureter and we checked ureter and renal pelvis and calyces by flexible ureteroscope along the guide-wire. If it is difficult to put the flexible ureteroscope in, we can do the ureteral expansion first.

\section{Results}

In 19 cases, we found urothelial carcinoma (postoperative pathology confirmed) 6 cases were located in the renal pelvis, 7 cases were located in calyces, and the other 6 cases were located in the upper of ureter, tumor size $4 \sim 7 \mathrm{~mm}$, average size was $5 \mathrm{~mm}$. 10 cases were calculi in upper of ureter, 8 cases were calculi in middle of ureter, 6 cases were nonspecific ureteropelvic inflammation, 8 cases werepelvic vein bleeding and in 9 cases we could not find disease. We took the electric coagulation hemostasis in 7 cases of pelvic vein bleeding, and took surgery to cure the 17 cases of urothelial carcinoma.Other 19 cases of calculi underwent the treatment to take the stone out. 6 patients with nonspecific ureteropelvic inflammation, took the third-generation cephalosporins treatment and recovered after $2 \sim 4$ weeks treatment. Surgery time of the 58 patients was 10 70 min, and average time was $40 \mathrm{~min}$; postoperative time in hospital was 3 9 days, average time was 4 days. 5 cases got slight backache, recovered after anti-infection reatment . After following up for $3 \sim 12$ months, 17 cases of urothelial carcinoma got regularly cystoscope, and no recurrence happened. The hematuresis of the 6 cases of nonspecific ureteropelvic inflammation disappeared. Urine routine in the 7 cases of pelvic vein hemorrhage were normal after operations in1- 2 months.

\section{Discussion}

With the development of endoscopic techniques, treating urinary tract diseases by ureteroscopy has become a very important clinical urology method. But with the characteristic of itself, it can only observe part of the ureter lesions. Flexible ureteroscope has the benefit that the lens can be bent $180^{\circ}$, so it is a valuable method. Especially in the examination and treatment of middle and upper of urinary tract lesion, it has a certain advantage [1-2]. It can be observed from different directions of ureteral segments, renal pelvis and calyces to clearly diagnose and treat the urinary tract diseases.

For the early renal pelvis and calyces and ureteral hematuria caused by a small lesions, it is often difficult to be found in a regular examination and clinical doctors can't make the positive diagnosis [3]. Most of these patients need to do this inspection. The tumor in calyx $<0.5 \mathrm{~cm}$, has a greater chance of misdiagnosis. The Flexible ureteroscope makes the part of the patients have an effective diagnosis and treatment. 17 cases of unexplained hematuria have been to several hospitals, because the tumor is small, there was not clear diagnosis. The application of flexible ureteroscope inspection on patients, found that renal pelvis tumor in 5 cases, renal calyx tumor 7 cases, the upper ureteral tumors in the 5 cases, the largest diameter was about $8 \mathrm{~mm}$, and minimum diameter was about $4 \mathrm{~mm}$. Confirmed by postoperative pathology for urothelial carcinoma made the patients receive timely diagnosis and treatment. In operation, flexible ureteroscope is much more difficult than ureteroscopy, for it is difficult to grasp the operation skills and also have different methods in operation [4].There is a kind of extreme bending ureteroscopy which can enter into the renal pelvis and be flexible ureteroscope operate freely, may greatly reduce the difficulty of learning [5].

HU W L and other people [6] think that in operation the first step is do the ureteroscopy.On the one hand it can expand ureter and openings, on the other hand can be a preliminary 
understanding of urethra, bladder and ureter. Before ureteroscopy exit placed 1 guide-wire at the same time, then along the guide-wire into the ureter expansion pipe and F12 ureter sheath to the ureter, retained ureter sheath and thread, makes the next step of flexible ureteroscope easier checking. Flexible ureteroscope has been more widely used in foreign countries, and the author has gradually master the technology of the use of flexible ureteroscope. According to the author's clinical experience, the majority of cases were under the guide-wire guide directly into flexible ureteroscope in the diagnosis and treatment for urinary tract diseases, and a few cases need to advance the expansion of the ureter. Using ureteroscopy can make a diagnosis and give treatment of the above parts, can also give new crushed stone gravel equipment, which greatly improve the success rate of the minimally invasive treatment of ureteral calculi [7].

Flexible ureteroscope make a diagnosis and give treatment of 58 cases, 19 cases of ureteral calculi patients come to the hospital due to the hematuresis.Because calculi was small, so the imaging failed to find the stone on the middle and upper period of ureter. Flexible ureteroscope is suitable for the treatment which ureteroscopy is unable to treat the calculi in the upper ureter, and the calculi which percutaneous nephroscope cannot be found within the upper and lower calyx [8]

The author thinks that flexible ureteroscope for upper tract lesions, especially in patients with unexplained hematuria, can clear diagnosis, and can get proper treatment. It is a reliable method in treatment of upper tract lumen small lesions, and it is worthy of clinical promotion.

\section{Reference}

[1] PA N H M, X U LP. A pplication of flexible ureteroscope in diagnosis and treatm ent of renal colic [J]. China Journal of Endoscopy, 2005, 11(10): 1041-1042. Chinese

[2] LUO H B, LIU X H, WU T P, et al. Minimally invasive percutaneous nephrostomy Nd: YAG laser lithotripsy with rigid ureteroscope combined with flexible ureteroscope for complex upper urinary calculi [J]. China Journal of Endoscopy, 2007, 7: 681-683.Chinese

[3] SHEN JH, NA Y Q, GAO Y L. Application of flexible ureteroscope in diagnosis of upper urinary tract disease (Report of 20 cases) [J]. Chin J U rol, 1996, 17(3): 170. Chinese

[4] SUN Y H, GAO X, GAO X F, et al. Holmium laser lithotripsy with flexible ureteroscope for calyceal calculi (Report of 78 cases) [J]. Journal of Clinical U rology, 2004, 19 (3): 139-141. Chinese

[5] SUN Y H, WANG LH, XU CL, et al. Preliminary experience in managing renal calyceal calculi with actively deflectable, flexible ureteroscopy [J]. Chin J Urol, 2005, 26(11): 743-745. Chinese

[6] HU W L, DENG ZX, ZHAO Y B, et al. Application of flexible ureteroscope in diagnosis and treatment of upper urinary tract disease $[\mathrm{J}]$. Chinese Journal of Minim ally Invasive Surgery, 2008, 8(4): 335-336. Chinese

[7] QI D F, ZENG G H, YUAN J, et al. Treatment of stones impacted in small inner diameter uretes with $6.9 \mathrm{~F}$ ureterscopy combined with holmium leaser [J]. China Journal of Endoscopy, 2008, 14(11): 1171-1173. Chinese 
[8] ZHANG M C, KONG X B, ZHANG G, et al. Treatment of complex upper urinary calculi with Holmium laser lithotriptor through flexible ureteroscope [J]. China Journal of Endoscopy, 2008, 14(8):810-811. Chinese 